\title{
Multicenter Phase II Study of Intravenous and Intraperitoneal Paclitaxel With S-1 for Pancreatic Ductal Adenocarcinoma Patients With Peritoneal Metastasis
}

\author{
Sohei Satoi, MD, ${ }^{*}$ Tsutomu Fujii, MD, † Hiroaki Yanagimoto, MD, ${ }^{*}$ Fuyuhiko Motoi, MD, $\ddagger$ \\ Masanao Kurata, MD, $\S$ Naminatsu Takahara, MD, Suguru Yamada, MD, $\dagger$ Tomohisa Yamamoto, MD, * \\ Masamichi Mizuma, MD, $\ddagger$ Goro Honda, MD,|| Hiroyuki Isayama, MD, $\uparrow$ Michiaki Unno, MD, $\ddagger$ \\ Yasuhiro Kodera, MD, $\dagger$ Hironori Ishigami, MD, ${ }^{* *}$ and Masanori Kon, MD
}

\begin{abstract}
Objective: To evaluate the clinical efficacy and tolerability of intravenous (i.v.) and intraperitoneal (i.p.) paclitaxel combined with S-1, "an oral fluoropyrimidine derivative containing tegafur, gimestat, and otastat potassium" in chemotherapy-naive pancreatic ductal adenocarcinoma (PDAC) patients with peritoneal metastasis.

Background: PDAC patients with peritoneal metastasis (peritoneal deposits and/or positive peritoneal cytology) have an extremely poor prognosis. An effective treatment strategy remains elusive.

Methods: Paclitaxel was administered i.v. at $50 \mathrm{mg} / \mathrm{m}^{2}$ and i.p. at $20 \mathrm{mg} / \mathrm{m}^{2}$ on days 1 and 8 . S- 1 was administered at $80 \mathrm{mg} / \mathrm{m}^{2} / \mathrm{d}$ for 14 consecutive days, followed by 7 days of rest. The primary endpoint was 1-year overall survival (OS) rate. The secondary endpoints were antitumor effect and safety (UMIN000009446).

Results: Thirty-three patients who were pathologically diagnosed with the presence of peritoneal dissemination $(n=22)$ and/or positive peritoneal cytology $(\mathrm{n}=11)$ without other organ metastasis were enrolled. The tumor was located at the pancreatic head in 7 patients and the body/tail in 26 patients. The median survival time was $16.3(11.47-22.57)$ months, and the 1-year survival rate was $62 \%$. The response rate and disease control rate in assessable patients were $36 \%$ and $82 \%$, respectively. OS in 8 patients who underwent
\end{abstract}

From the *Department of Surgery, Kansai Medical University; †Department of Gastroenterological Surgery (Surgery II), Nagoya University Graduate School of Medicine; †Department of Surgery, Tohoku University Graduate School of Medicine; §Department of Surgery, University of Tsukuba; \Department of Gastroenterology, Graduate School of Medicine, the University of Tokyo; |Department of Surgery, Tokyo Metropolitan Cancer and Infectious Diseases Center Komagome Hospital; and ${ }^{* *}$ Department of Chemotherapy, the University of Tokyo.

SS and TF contributed equally to this study.

Authors' contributions: SS, TF, and MK contributed to all aspects of this study and article. HY, FM, GH, HI, and HI contributed to study conception and design, experiments, collection of the data, and critical revision of the article. MK, NT, SY, TY, MM, MU, and YK contributed to collection of the data and critical revision of the article. All authors approved the final draft of the article.

Funding: This study was financially supported by the Japanese Foundation for Multidisciplinary Treatment of Cancer (http://www.ifmc.or.ip/).

Conflicts of interest: In addition, conflicts of interest of the authors were listed. Professor Kodera is supported by grants from the Taiho Pharmaceutical Company and Bristol Myers Squib. Professor Unno is supported by grants from the Chugai Pharmaceutical Company, Yakult Honsha, Janssen Pharmaceutical Company and Takeda Pharmaceutical Company. Professor Isayama is supported by a grant from the Taiho Pharmaceutical Company.

This is an open-access article distributed under the terms of the Creative Commons Attribution-Non Commercial-No Derivatives License 4.0, where it is permissible to download and share the work provided it is properly cited. The work cannot be changed in any way or used commercially.

Reprints: Sohei Satoi, MD, FACS. Department of Surgery, Kansai Medical University, 2-5-1, Shin-machi, Hirakata 573-1010, Japan.

E-mail: satoi@ hirakata.kmu.ac.ip.

Copyright (C) 2016 Wolters Kluwer Health, Inc. All rights reserved.

ISSN: 0003-4932/16/26502-0397

DOI: $10.1097 /$ SLA.0000000000001705 conversion surgery was significantly higher than that of nonsurgical patients ( $\mathrm{n}=25, P=0.0062)$. Grade $3 / 4$ hematologic toxicities occurred in $42 \%$ of the patients and nonhematologic adverse events in $18 \%$. One patient died of thrombosis in the superior mesenteric artery.

Conclusions: This regimen has shown promising clinical efficacy with acceptable tolerability in chemotherapy-naive PDAC patients with peritoneal metastasis.

Keywords: intraperitoneal chemotherapy, paclitaxel, pancreatic ductal adenocarcinoma, peritoneal metastasis, S-1

(Ann Surg 2017;265:397-401)

$P$ ancreatic ductal adenocarcinoma (PDAC) continues to have a dismal prognosis with a 5-year survival rate of $<5 \%$ even in the modern era. ${ }^{1,2}$ The median survival time (MST) of patients with distant organ metastasis, including peritoneal metastasis, ${ }^{3}$ is extremely poor at less than 12 months. Moreover, the presence of peritoneal metastasis is associated with development of intestinal obstruction, massive ascites, and malnutrition, leading to poor performance status, ${ }^{4}$ which, in turn, deprives the patients of the opportunity to receive chemotherapy. ${ }^{5}$ MST of these patients has been reported to be 6 weeks from a population-based study in the Netherlands, ${ }^{3}$ and 7 weeks in another series of 73 patients with malignant ascites. ${ }^{4}$ Pharmacokinetic studies revealed that anticancer drugs administered systemically do not necessarily enter the peritoneal cavity. Compared with systemic chemotherapy, intraperitoneal (i.p.) chemotherapy seems to be advantageous for treatment of peritoneal dissemination due to a high drug concentration in the peritoneal cavity to directly contact tumor nodules. ${ }^{6-10}$

The clinical effects of i.p. paclitaxel (PTX) in patients with peritoneal metastasis have been favorably reported in clinical trials for ovarian cancer, ${ }^{6,7}$ gastric cancer, ${ }^{8,9}$ and even PDAC. ${ }^{10}$ Most notably, Ishigami et $\mathrm{al}^{8}$ conducted a phase II study of weekly intravenous (i.v.) and i.p. PTX with S-1 in gastric cancer with peritoneal metastases, with remarkable results such as overall response rate (ORR) of $56 \%$, disappearance or marked decrease in malignant ascites in $62 \%$, and 1 -year overall survival (OS) rate of $78 \%$. Kamei et al ${ }^{11}$ demonstrated that i.p. administration of PTX nanoparticles resulted in high accumulation in disseminated nodules, presumably due to its superior penetrating activity directly into malignant tissue in a mouse. Thus, i.p. chemotherapy using PTX is considered to be an ideal therapeutic approach for peritoneal carcinomatosis from the viewpoint of drug delivery. In addition, S-1 is an oral fluoropyrimidine derivative which significantly prevented peritoneal carcinomatosis in gastric cancer in the postoperative adjuvant setting, and has shown efficacy also in the treatment of pancreatic cancer.

Therefore, we have conducted a phase II study in a multicenter setting to evaluate the clinical efficacy and tolerability of i.v. and i.p. 
PTX combined with $S-1^{8}$ in PDAC patients with peritoneal metastasis, but without other distant organ metastases.

\section{METHODS}

\section{Patient Recruitment}

From December 2012 to March 2015, 33 patients were enrolled in this phase II study at 7 Japanese centers. The eligibility criteria were as follows: histologically proven PDAC; presence of cancer cells on peritoneal cytology performed using staging laparoscopy in patients with radiographically defined unresectable locally advanced PDAC, or peritoneal dissemination in all types of PDAC on staging laparoscopy or open laparotomy; chemotherapy-naive; Eastern Cooperative Oncology Group performance status 0 to 1 ; adequate bone marrow function (leukocyte count $3500-12,000 / \mathrm{mm}^{3}$, hemoglobin $>8.0 \mathrm{~g} / \mathrm{dL}$, and platelet count $>100,000 / \mathrm{mm}^{3}$ ); adequate liver function (serum total bilirubin $<2.0 \mathrm{mg} / \mathrm{dL}$ and serum transaminases $<150 \mathrm{IU} / \mathrm{L}$ ); adequate renal function (serum creatinine $<1.2 \mathrm{mg} / \mathrm{dL}$ ); age $>20$ years and $<80$ years. The exclusion criteria were as follows: presence of metastasis in other distant organs such as the liver, lungs, bone or others, positive peritoneal washing cytology in patients with resectable or borderline resectable PDAC; other active concomitant malignancies; other severe medical conditions. Written informed consent was obtained from all patients. This study was conducted in accordance with the Declaration of Helsinki, and the study protocol was approved by the institutional review board of the affiliated hospital. The registration number of this clinical trial is UMIN000009446.

\section{Treatment}

On diagnosing peritoneal dissemination or positive peritoneal cytology during staging laparoscopy or open laparotomy, a peritoneal access port was implanted in the lower abdomen, with a catheter placed in the pelvic cavity. S-1 was administered orally twice daily at a dose of $80 \mathrm{mg} / \mathrm{m}^{2} / \mathrm{d}$ for 14 consecutive days, followed by 7 days of rest. PTX was administered i.v. at a dose of $50 \mathrm{mg} / \mathrm{m}^{2}$ and i.p. at $20 \mathrm{mg} / \mathrm{m}^{2}$ on days 1 and 8 . These dosages had been determined by a phase I study in gastric cancer, ${ }^{22}$ and the safety at the same dosages was confirmed in a feasibility study involving 6 patients with peritoneal metastasis from pancreatic cancer. PTX was diluted in $1 \mathrm{~L}$ of normal saline and administered through the implanted peritoneal access port over 1 hour concurrently with i.v. infusion after standard premedication. The treatment course was repeated every 3 weeks until observation of unacceptable toxicity, disease progression, or surgery. Surgical resection (conversion surgery) was performed at a discretion of the surgeons when exceptional response to the chemotherapy was observed. Although the criteria for conversion to surgery had not been prespecified in the protocol, there had been a consensus among the participating investigators that a patient who fulfilled all of the following without deterioration in the performance status could be indicated for surgical resection: tumor remission was observed by the contrast-enhanced computed tomography $(\mathrm{CT})$ in case it had been unresectable locally advanced tumor, tumor markers decreased, peritoneal washing cytology turned negative in case it had been positive, and peritoneal deposits became invisible by the staging laparoscopy in case it had been observed at the time of inclusion into the trial. Although there were no strict rules regarding the number of courses or the duration of chemotherapy to be given before surgery, our previous finding that the interval longer than 8 months between the initial treatment and surgical resection was associated with favorable prognosis in PDAC patients with initially unresectable disease ${ }^{23}$ had been shared by the investigators.

\section{Primary and Secondary Endpoints}

The primary endpoint was 1-year OS rate, and the secondary endpoints were ORR, frequency of negative peritoneal washing cytology, ascites-onset rate within 1 year after initial treatment, resection rate, and safety. Objective tumor responses were evaluated every 2 months during the study, and classified based on the Response Evaluation Criteria in Solid Tumors (RECIST) guidelines. ${ }^{12}$ To evaluate the antitumor effects on peritoneal metastases, peritoneal washing cytology through a peritoneal access port was examined using Papanicolaou and May-Giemsa staining every 2 months. Toxicity was monitored weekly and graded according to the National Cancer Institute-Common Terminology Criteria for Adverse Events version 4.0.

\section{Statistical Analysis}

The sample size was calculated as follows. An estimated 1 -year survival rate of patients with metastatic PDAC was $25 \%$. Assuming a null hypothesis of $25 \%$ and an alternative hypothesis of $45 \%$ with 1-sided type I error of 0.05 and power of 0.8 , with an accrual time of 2 years and follow-up of 1 year after closure of recruitment, enrollment of 24 patients was required. Continuous variables were expressed as median and range. The MST was estimated using the Kaplan-Meier method. Patients alive at the time of follow-up were censored. The last follow-up date was December 2015. Statistical analyses were performed using JMP statistical discovery software (JMP version 11.0, SAS Institute, Cary, NC). A $P$ value $<0.05$ was considered statistically significant.

\section{RESULTS}

\section{Clinical Background}

As shown in Table 1, 33 patients were enrolled, including 22 patients with peritoneal dissemination (of which peritoneal washing cytology was positive in 21 patients), and 11 patients with positive peritoneal washing cytology. Malignant ascites was observed in 15 patients on laparoscopy or laparotomy. Primary tumors were categorized as unresectable locally advanced disease in 19 patients, borderline resectable in 3 patients, and resectable in 11 patients, respectively. Median age was 69 years (range 42-79), and the maleto-female ratio was 14:19. The tumor was located at the pancreatic head in 7 patients and the body/tail in 26 patients, and the median diameter of the tumor was $40(22-105) \mathrm{mm}$. The median carbohydrate antigen 19-9 (CA19-9) level was $344(1-25,850) \mathrm{U} / \mathrm{mL}$ at the baseline. Performance status was 0 in 20 patients and 1 in 13 patients. Five patients underwent biliary drainage due to obstructive jaundice before the enrollment.

\section{TABLE 1. Patient Characteristics}

\begin{tabular}{lc}
\hline Parameters & $\begin{array}{c}\text { All Eligible Patients } \\
(\mathbf{n}=\mathbf{3 3})\end{array}$ \\
\hline Age (range), y & $69(42-79)$ \\
Male:female & $14: 19$ \\
Body mass index (range) & $21.42(13.34-24.17)$ \\
Performance status (\%), 0:1 & $20(61 \%): 13(39 \%)$ \\
Tumor location, head:body/tail & $7: 26$ \\
Tumor diameter (range), mm & $40(22-105)$ \\
Resectable: borderline resectable: unresectable* & $11: 3: 19$ \\
Ascites, -:+ & $18: 15(45 \%)$ \\
Peritoneal dissemination, -:+ & $11: 22(67 \%)$ \\
Peritoneal (washing) cytology, -:+ & $1: 32(97 \%)$ \\
Albumin (range), g/L & $3.9(2.7-4.3)$ \\
CA19-9 (range), U/mL & $344(1-25850)$ \\
Duration of protocol therapy & $8.8(0.8-22.6)$ \\
$\quad$ (range), mo &
\end{tabular}

The data are expressed as median and range.

*Resectability status was defined according to the NCCN guideline. 


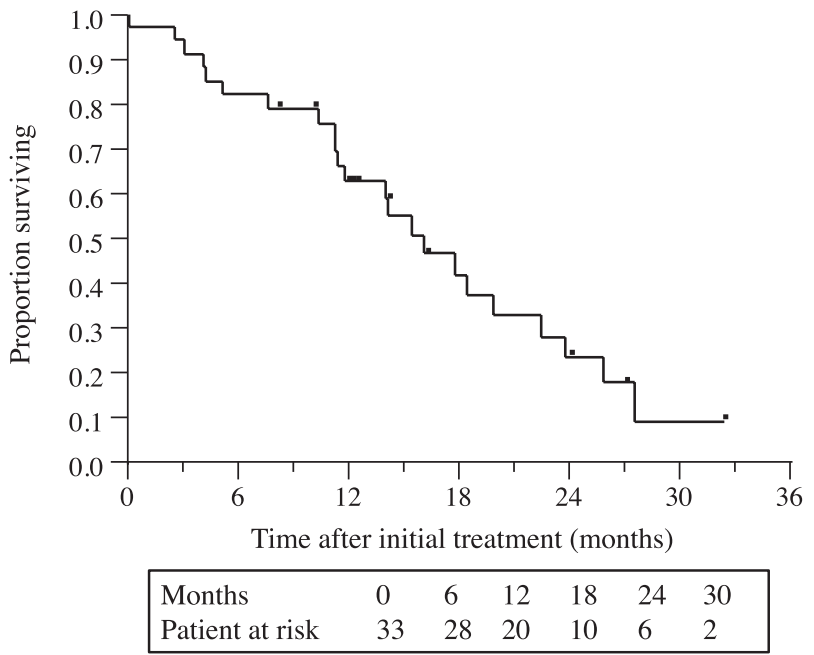

FIGURE 1. Overall survival curve of 33 PDAC patients with peritoneal metastasis. The median survival time was 16.3 months, and 1- and 2-year survival rates were $62 \%$ and $23 \%$, respectively.

\section{Survival and Objective Response}

This regimen was administered for a median of $8.8(0.8-22.6)$ months. As shown in Figure 1, 23 out of 33 patients have already died, with a median observation period of 13.4 (8.2-32.6) months or until death. Thirty-one out of 33 patients were followed up for at least 12 months. The MST was 16.3 (11.47-22.57) months, and 1 and 2-year OS rates were $62 \%$ and $23 \%$, respectively (Fig. 1). As shown in Table 2, the ORR by RECIST criteria was $36 \%$ and the disease control rate was $82 \%$. Peritoneal washing cytology turned negative in 18 of 33 patients $(55 \%)$. During treatment, the median rate of CA19-9 decrease was $51 \%$, and normalization of CA19-9 was observed in $35 \%$ of patients. Malignant ascites was observed in 15 of 33 patients at diagnosis of peritoneal metastasis. During treatment, ascites disappeared in 9 of 15 patients within 1 year of initial treatment; the remaining 6 patients currently have malignant ascites. Another 4 patients newly developed malignant ascites. Overall, 10 out of 33 patients (30\%) had malignant ascites within 1 year of initial treatment.

\section{Conversion Surgery}

Of the 33 patients, 8 patients $(24 \%)$ including 5 patients who had peritoneal dissemination and 3 patients with positive peritoneal washing cytology status plus unresectable locally advanced cancer

\section{TABLE 2. Clinical Responses}

\begin{tabular}{lc}
\hline Parameters & $\begin{array}{c}\text { All Eligible } \\
\text { Patients }(\mathbf{n}=\mathbf{3 3})\end{array}$ \\
\hline Minimum value of CA19-9 (range), U/mL & $30.2(0.7-6.548)$ \\
Decreased rate of CA19-9 (range), $\%$ & $51(-59.3-99.7)$ \\
Normalization of CA19-9, \% & $9 / 26(35)$ \\
Tumor response & \\
$\quad$ Complete response, \% & $0(0)$ \\
Partial response, \% & $12(36)$ \\
Stable disease, \% & $15(46)$ \\
Progressive disease, $\%$ & $2(6)$ \\
Not evaluated, \% & $4(12)$ \\
Peritoneal cytology, turned negative, $\%$ & $18(55)$ \\
\hline
\end{tabular}

underwent pancreatectomy (Table 3). All patients underwent surgical resection more than 8 months after the initiation of chemotherapy. Distal pancreatectomy with celiac axis resection was performed in 2 patients, radical antegrade modulated pancreatosplenectomy was performed in 2 patients, distal pancreatectomy was performed in 2 patients, total pancreatectomy with portal vein resection was performed in 1 patient, and pancreatoduodenectomy with portal vein resection in 1 patient. Five out of 8 patients underwent concomitant major artery and/or portal vein resection. The consequences were R0 resection in 6 patients and $\mathrm{R} 1$ resection in 2 patients. No in-hospital deaths occurred in patients who underwent surgical resection. Pathological staging revealed T3N1M0 in 6 patients and T3NOM0 in 2 patients. Evans classification ${ }^{13}$ was IIA in 6 patients and IIB in 2 patients. As shown in Figure 2, OS of patients who underwent conversion surgery was significantly better than that of patients who did not undergo conversion surgery $(P=0.0038)$, and MST was also longer (27.8 vs $14.2 \mathrm{mo}$, respectively).

\section{Adverse Event Profile}

Overall results are listed in Table 4, grade 3/4 hematologic adverse events included neutropenia (42\%), leukopenia (18\%), febrile neutropenia (6\%), and anemia (3\%). Grade 3/4 nonhematologic adverse events included appetite loss in $12 \%$, nausea in $9 \%$, vomiting and diarrhea in $6 \%$, and mucositis in $6 \%$. A total of 3 patients discontinued treatment after 1 month. One of these patients died of superior mesenteric arterial thrombosis after the first infusion of this regimen, and this case was regarded as a treatment-related death. Other severe adverse events were anaphylactic reaction during the first infusion of this regimen and severe mucositis and diarrhea. Complications related to the peritoneal access device presented as infection of the i.p. catheter in 1 patient, and dislocation of the device in 2 patients.

\section{DISCUSSION}

Recent progress in chemotherapy has provided an improved prognosis in patients with unresectable PDAC. ${ }^{14,16}$ In particular, MST of patients with metastatic disease has increased up to 11.5 months with the FOLFIRINOX regimen ${ }^{15}$ and 8.5 months with the gemcitabine + nab-PTX regimen. ${ }^{14}$ ORR of FOLFIRINOX (oxaliplatin, irinotecan, 5-FU, and leucovorin) and gemcitabine + nab-PTX regimen was $31 \%{ }^{15}$ and $23 \%,{ }^{14}$ respectively. Given the pharmacokinetics of intravenously administered agent, it remains doubtful whether these advances in systemic treatment also translate into improvements in the outcome of patients with peritoneal metastasis. In reality, prognosis of PDAC patients with peritoneal metastasis remains extremely poor (MST 6-7 wks). ${ }^{3,4,17-19}$

A standard chemotherapy regimen for unresectable PDAC in Japan is S-1 (MST 9.7 mo), survival of which was revealed to be noninferior to that of gemcitabine (MST $8.8 \mathrm{mo}$ ) in the GEST study (randomized phase III study of Gemcitabine plus S-1, S-1 alone, or Gemcitabine alone in patients with locally advanced and metastatic pancreatic cancer). ${ }^{16} \mathrm{~A}$ few authors reported i.v. PTX as a potential treatment option in patients with gemcitabine-refractory pancreatic cancer. $^{20-22}$ Ishigami et al ${ }^{8,23}$ established i.v./i.p. PTX + S-1 therapy in gastric cancer patients with peritoneal metastasis in a phase I/II study. These investigators suggested that i.p. administration of anticancer drugs enabled an extremely high concentration of drugs to directly contact the target cancer lesions in the peritoneal cavity due to its large molecular weight and fat solubility. ${ }^{8,23}$

The present study focused on the clinical efficacy and feasibility of i.v./i.p. PTX + S-1 therapy in chemotherapy-naive patients with peritoneal metastasis without metastasis in other distant sites. The MST was 16.3 months (11.47-22.57), and the 1-year OS rate was $62 \%$. Ferrone et al ${ }^{18}$ reported an MST of 7 months in PDAC 


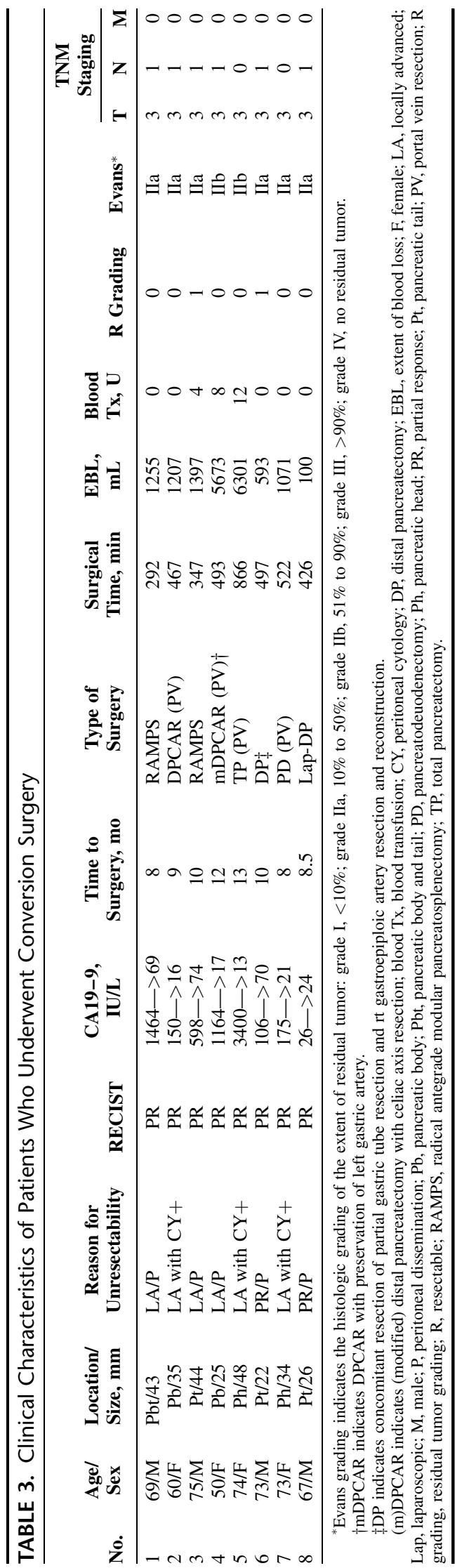

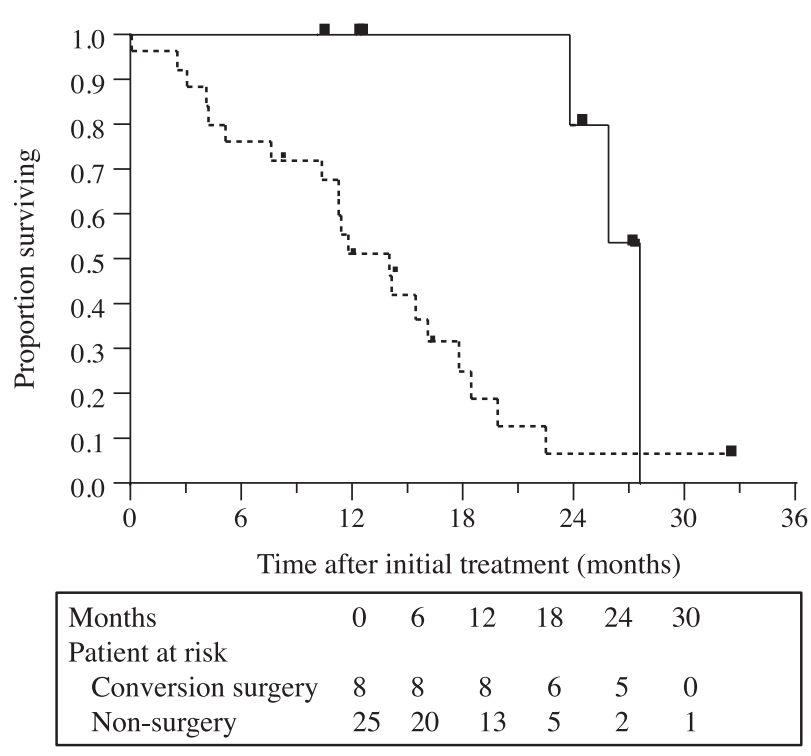

FIGURE 2. Comparison of survival curves between patients who underwent conversion surgery ( $n=8$, solid line) and no surgery ( $\mathrm{n}=25$, broken line). Overall survival of patients who underwent conversion surgery was significantly longer than that of patients without conversion surgery $(P=0.0038)$, and MST was 27.8 months in the former group and 14.2 months in the latter group.

patients with peritoneal dissemination, and 6 months in patients with locally advanced disease who had positive peritoneal washing cytology during staging laparoscopy. Our previous report also revealed that ascites occurred within 1-year after initiation of chemotherapy in approximately $70 \%$ of patients with peritoneal metastasis diagnosed by staging laparoscopy for radiographically defined locally advanced PDAC. ${ }^{5}$ Considering that the patients with peritoneal metastasis generally have a particularly poor prognosis, the present survival results are encouraging.

In the current study, the high response and disease control rates (36\% and $82 \%$, respectively) in addition to the efficacy in eliminating peritoneal deposits and intraperitoneal free cancer cells allowed surgeons to perform conversion surgery in selected patients. Surprisingly, the rate of conversion surgery was $24 \%$ in this study, and pathological response of some extent was observed in all patients who underwent conversion surgery. Moreover, the MST in patients who underwent conversion surgery was 27.8 months, which was comparable to that in patients with resectable PDAC. The MST after conversion surgery for PDAC has actually been reported to reach 30 to 52 months. ${ }^{24-28}$ Unfortunately, only a small fraction of PDAC is eligible for conversion surgery, and in our experience, only 13 of 130 patients $(10 \%)$ with initially unresectable locally advanced PDAC underwent surgical resection after a favorable response to chemo(radio)therapy. ${ }^{28}$ Thus, the i.v./i.p. PTX + S-1 combination has shown remarkable performance, both in terms of the conversion rate and outcome of patient who received conversion surgery. This regimen therefore has the potential to control not only peritoneal metastasis but also the primary tumor.

Although 1 treatment-related death due to thrombosis in the superior mesenteric artery and 1 anaphylactic reaction were observed, most of the adverse events observed in this study were similar to those seen in gastric cancer patients with peritoneal metastasis. ${ }^{8}$ The median duration of treatment was 8.8 months in the present study, which was relatively longer than the 2.6 to 4.3 
TABLE 4. Profile of Adverse Events

\begin{tabular}{lrrrrr}
\hline Grading by CTCAEv4.0 & $\mathbf{1}$ & $\mathbf{2}$ & $\mathbf{3}$ & $\mathbf{4}$ & $\mathbf{3 / 4}$ \\
\hline Leucocytopenia & 5 & 10 & 4 & 2 & $18 \%$ \\
Neutropenia & 0 & 4 & 8 & 6 & $42 \%$ \\
Febrile neutropenia & 0 & 0 & 1 & 1 & $6 \%$ \\
Anemia & 12 & 11 & 1 & 0 & $3 \%$ \\
Thrombocytop enia & 4 & 2 & 1 & 1 & $6 \%$ \\
Liver function & 10 & 1 & 0 & 0 & $0 \%$ \\
General fatigue & 5 & 2 & 1 & 0 & $3 \%$ \\
Appetite loss & 10 & 3 & 4 & 0 & $12 \%$ \\
Nausea & 7 & 1 & 3 & 0 & $9 \%$ \\
Vomiting & 1 & 1 & 2 & 0 & $6 \%$ \\
Diarrhea & 5 & 1 & 2 & 0 & $6 \%$ \\
Skin rash & 4 & 1 & 0 & 0 & $0 \%$ \\
Mucositis oral (stomatitis) & 5 & 1 & 2 & 0 & $6 \%$ \\
Peripheral neuropathy & 6 & 2 & 0 & 0 & $0 \%$ \\
Alopecia & 14 & 4 & 0 & 0 & $0 \%$ \\
Edema & 2 & 1 & 0 & 0 & $0 \%$ \\
Pneumonia & 1 & 1 & 0 & 0 & $0 \%$ \\
Dysgeusia & 8 & 0 & 0 & 0 & $0 \%$ \\
Anaphylaxis & 0 & 0 & 0 & 1 & $3 \%$ \\
\hline
\end{tabular}

months previously reported in the GEST study. ${ }^{16}$ Taken together, the i.v./i.p. PTX + S-1 combination was considered relatively safe and feasible.

Even with these highly promising results, this study can only be hypothesis-generating at this time, given the small sample size and the nonrandomized nature. Sustainable efforts are warranted to conduct a decently designed randomized clinical trial to confirm efficacy of this combination in the subset of PDAC patients with high risk of death due to the peritoneal disease.

In conclusion, i.v./i.p. PTX + S-1 provides promising and encouraging clinical efficacy and acceptable tolerability in chemotherapy-naive PDAC patients with peritoneal metastasis.

\section{ACKNOWLEDGMENTS}

We would like to express our sincere appreciation to Drs $S$. Yamaki, S. Hirooka, H. Ryota, N. Kondo, Y. Murakami, Y. Nagakawa, A. Tsuchida, A. Matsushita, Y. Nakamura, K. Asai, M. Watanabe, N. Sato, S. Hirano, K. Wada, M. Yasunaga, S. Shimizu, F. Miura, and N. Ikeda in the Japan study group of pancreatic ductal adenocarcinoma with peritoneal metastasis for their significant contribution to this study.

\section{REFERENCES}

1. Egawa S, Toma H, Ohigashi H, et al. Japan Pancreatic Cancer Registry; 30th year anniversary: Japan Pancreas Society. Pancreas. 2012;41:985-992.

2. Ma J, Siegel R, Jemal A. Pancreatic cancer death rates by race among US men and women, 1970-2009. J Natl Cancer Inst. 2013;105:1694-1700.

3. Thomassen I, Lemmens VE, Nienhuijs SW, et al. Incidence, prognosis, and possible treatment strategies of peritoneal carcinomatosis of pancreatic origin: a population-based study. Pancreas. 2013;42:72-75.

4. Takahara N, Isayama H, Nakai Y, et al. Pancreatic cancer with malignant ascites. Pancreas. 2015;44:380-385.

5. Satoi S, Yanagimoto $\mathrm{H}$, Yamamoto $\mathrm{T}$, et al. A clinical role of staging laparoscopy in patients with radiographically defined locally advanced pancreatic cancer. World J Surg Oncol. 2016;14:14.

6. Armstrong DK, Bundy B, Wenzel L, et al. Intraperitoneal cisplatin and paclitaxel in ovarian cancer. $N$ Engl J Med. 2006;354:34-43.

7. Markman M, Brady MF, Spirtos NM, et al. Phase II trial of intraperitoneal paclitaxel in carcinoma of the ovary, tube, and peritoneum: a Gynecologic Oncology Group Study. J Clin Oncol. 1998;16:2620-2624.
8. Ishigami H, Kitayama J, Kaisaki S, et al. Phase II study of weekly intravenous and intraperitoneal paclitaxel combined with S-1 for advanced gastric cancer with peritoneal metastasis. Ann Oncol. 2010;21:67-70.

9. Kodera Y, Imano M, Yoshikawa T, et al. A randomized phase II trial to test the efficacy of intra-peritoneal paclitaxel for gastric cancer with high risk for the peritoneal metastasis (INPACT trial). Jpn J Clin Oncol. 2011;41:283286.

10. Takahara N, Isayama $\mathrm{H}$, Nakai $\mathrm{Y}$, et al. Intravenous and intraperitoneal paclitaxel with S-1 for refractory pancreatic cancer with malignant ascites: an interim analysis. J Gastrointest Cancer. 2014;45:307-311.

11. Kamei T, Kitayama J, Yamaguchi H, et al. Spatial distribution of intraperitoneally administrated paclitaxel nanoparticles solubilized with poly (2methacryloxyethyl phosphorylcholine-co n-butyl methacrylate) in peritoneal metastatic nodules. Cancer Sci. 2011;102:200-205.

12. Therasse P, Arbuck SG, Eisenhauer EA, et al. New guidelines to evaluate the response to treatment in solid tumors. European Organization for Research and Treatment of Cancer, National Cancer Institute of the United States, National Cancer Institute of Canada. J Natl Cancer Inst. 2000;92: 205-216.

13. Evans DB, Rich TA, Byrd DR, et al. Preoperative chemoradiation and pancreaticoduodenectomy for adenocarcinoma of the pancreas. Arch Surg. 1992; $127: 1335-1339$.

14. Von Hoff DD, Ervin T, Arena FP, et al. Increased survival in pancreatic cancer with nab-paclitaxel plus gemcitabine. N Engl J Med. 2013;369:16911703. 31.

15. Conroy T, Desseigne F, Ychou M, et al. FOLFIRINOX versus gemcitabine for metastatic pancreatic cancer. N Engl J Med. 2011;364:1817-1825.

16. Ueno H, Ioka T, Ikeda M, et al. Randomized phase III study of gemcitabine plus $\mathrm{S}-1, \mathrm{~S}-1$ alone, or gemcitabine alone in patients with locally advanced and metastatic pancreatic cancer in Japan and Taiwan: GEST study. J Clin Oncol. 2013;31:1640-1648.

17. Clark CJ, Traverso LW. Positive peritoneal lavage cytology is a predictor of worse survival in locally advanced pancreatic cancer. Am J Surg. 2010;199:657-662.

18. Ferrone CR, Haas B, Tang L, et al. The influence of positive peritoneal cytology on survival in patients with pancreatic adenocarcinoma. $J$ Gastrointest Surg. 2006;10:1347-1353.

19. Jo JH, Chung MJ, Park JY, et al. Clinical characteristics of long-term survivors of inoperable pancreatic cancer. An 8-year cohort analysis in Korea. Pancreas. 2015;43:1022-1031.

20. Kim YJ, Bang S, Park JY, et al. Phase II study of 5-fluorouracil and paclitaxel in patients with gemcitabine-refractory pancreatic cancer. Cancer Chemother Pharmacol. 2009;63:529-533.

21. Maeda S, Motoi F, Onogawa T, et al. Paclitaxel as second-line chemotherapy in patients with gemcitabine-refractory pancreatic cancer: a retrospective study. Int J Clin Oncol. 2011;16:539-545.

22. Shukuya T, Yasui $\mathrm{H}$, Boku N, et al. Weekly paclitaxel after failure of gemcitabine in pancreatic cancer patients with malignant ascites: a retrospective study. Jpn J Clin Oncol. 2010;40:1135-1138.

23. Ishigami H, Kitayama J, Otani K, et al. Phase I pharmacokinetic study of weekly intravenous and intraperitoneal paclitaxel combined with S-1 for advanced gastric cancer. Oncology. 2009;76:311-314.

24. Satoi S, Yamaue H, Kato K, et al. Role of adjuvant surgery for patients with initially unresectable pancreatic cancer with a long-term favorable response to non-surgical anti-cancer treatments: results of a project study for pancreatic surgery by the Japanese Society of Hepato-Biliary-Pancreatic Surgery. $J$ Hepatobiliary Pancreat Sci. 2013;20:590-600.

25. Bickenbach KA, Gonen M, Tang LH, et al. Downstaging in pancreatic cancer: a matched analysis of patients resected following systemic treatment of initially locally unresectable disease. Ann Surg Oncol. 2012;19:1663-1669.

26. Donahue TR, Isacoff WH, Hines OJ, et al. Downstaging chemotherapy and alteration in the classic computed tomography/magnetic resonance imaging signs of vascular involvement in patients with pancreaticobiliary malignant tumors. Influence on patient selection for surgery. Arch Surg. 2011;146:836-843.

27. Katz MH, Fleming JB, Bhosale P, et al. Response of borderline resectable pancreatic cancer to neoadjuvant therapy is not reflected by radiographic indicators. Cancer. 2012;118:5749-5756.

28. Opendro SS, Satoi S, Yamamoto T, et al. Role of adjuvant surgery in initially unresectable pancreatic cancer after long-term chemotherapy or chemoradiation therapy: survival benefit? J Hepatobiliary Pancreat Sci. 2014;21:695-702. 